\title{
Bootstrap approach for conformance assessment of measurement processes
}

\author{
R. Schmitt, P. Fritz ${ }^{\star}$, and J. Lose \\ Laboratory for Machine Tools and Production Engineering RWTH Aachen University, Steinbachstraße 19, \\ Aachen, 52074, Germany
}

Received: 5 August 2010 / Accepted: 20 August 2010

\begin{abstract}
This paper proposes a bootstrap approach to determine the measurement uncertainty of measurement devices with unknown measurement equation (in this work, a structured light projection system is applied for the measurement of pull broaches) by use of workpieces that are calibrated by a coordinate measuring machine (CMM). The obtained uncertainty is used to perform a conformance assessment of the initial measurement process according to ISO 14253-1 and VDA 5.
\end{abstract}

Keywords: Measurement uncertainty; CMM; conformance assessment

\section{Introduction}

Full control of measuring processes is an essential requirement for combining assured product quality with costeffective production. The management of measuring processes and the need to prove their capability are described in standards such as ISO 9000ff [1,2], ISO 10012 [3] and ISO/TS 16949 [4]. Since too inaccurate measurement systems yield an additional decrease in process capability and hence constrain given tolerances, there has been continuing development of standards and guidelines for using test methods in companies in recent years, especially in the automotive sector in the US and Germany. Therefore, it is not surprising that the two main industrial guidelines for conformance assessment of measurement processes - that are the measurement system analysis (MSA) [5], developed by Ford, Chrysler and GM as well as the German guideline VDA $5[6]$ - are originated from there.

The scientific approach for conformance assessment of measurement processes is the estimation of the measurement uncertainty according to the Guide to the Expression of Uncertainty in Measurement (GUM) [7] in combination with decisions rules that are derived from ISO 14253-1 [8]. (A summary of standards and guidelines can be found in Tab. 1).

A special challenge is the proof of capability of complex processes, as the process is virtually unmanageable with the aid of conventionally applied standards. This work provides a basis of the conformant assessment of a structured light 3D-scanner that is used for measuring pull broaches. A main difficulty of this task is that there exists no known closed-form measurement equation. This

\footnotetext{
^ Correspondence: p.fritz@wzl.rwth-aachen.de
}

problem is quite common for optical measurement devices (e.g. laser trackers $[9,10])$.

Therefore, there is a need for a method of estimating measurement uncertainty that will allow the suitability even of complex processes to be tested with acceptable effort. A promising approach is to estimate the measurement uncertainty by means of calibrated workpieces according to ISO 15330-3 [11]. For this, a master workpiece of the measured object has to be built and to be calibrated. The masterpiece is then measured multiple times with the target measurement system - that is the structured light scanner - and the calibration uncertainties and biases are propagated to form a measurement uncertainty for the measurement system under test.

\section{Task}

The work that is presented in this paper is part of a project whose goal is to apply new measurement systems to the measurement of pull broaches (Fig. 1). Standard measurement systems that have been used for this task until now are either tactile a CMM, which needs laborious programming, long testing times and is hard to integrate into an in-process measurement station. Alternative approaches that follow attributive measurements such as gauging, are cost intensive, inflexible and do not come with a measurement uncertainty that can be easily used for conformance assessment.

The assignment for an alternative measurement system followed according to the IDENT method that was developed at WZL [12] and proposes the use of a structuredlight 3D scanner, because of its short testing time and cost effectiveness with sufficient accuracy. 
Table 1. Normative environment of conformance assessment.

\begin{tabular}{ccc}
\hline & ISO standards & Automotive guidelines \\
\hline $\begin{array}{c}\text { Quality management } \\
\text { systems }\end{array}$ & ISO 9000 ff. & ISO/TS 16949 \\
Measurement systems & ISO 10012 & (VDA 6.1, QS 9000) \\
Measurement uncertainty & ISO 13005 (GUM + GUMS1) & VDA 5 (2003) \\
Decision rules & ISO 15530-3 & MSA \\
\hline
\end{tabular}

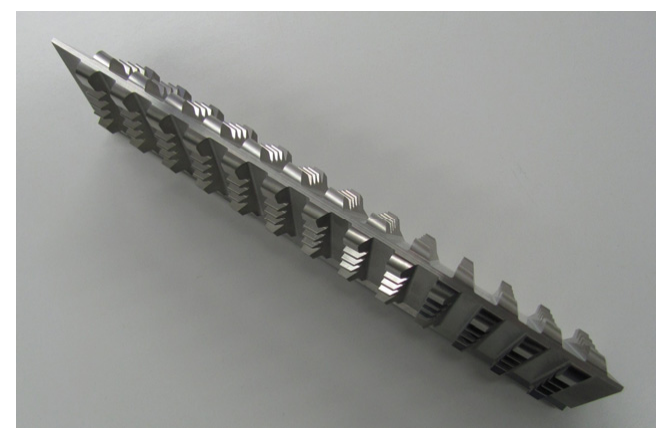

Fig. 1. Broach that has to be measured.

In phase 7 of the IDENT method, the assignment of measurement task and measurement system has to be validated, which means in this case that a conformant assessment of the structured-light 3D scanner has to be done.

As mentioned previously, as there exist no closedform measurement equation, other methods than standard GUM have to be applied to determine the measurement uncertainty. Since the industrial-based MSA and VDA 5 basically compute means of deviation only by repeated measurements, they do not include an accurate modeling of uncertainty components that cannot be observed such as temperature or measurement strategy.

A solution to this problem is to determine the measurement uncertainty via a calibrated workpiece according to ISO 15530-3. To determine the measurement uncertainties according to ISO 15530-3, a workpiece with defined similarity properties has to be calibrated: in this work, a precise lab CMM is used for calibration. The calibration uncertainties in turn are calculated by using a well known measurement model of the CMM.

Two methods are common to estimate uncertainties of CMMs: the most well known is the virtual CMM developed by a consortium lead by PTB [13]. The virtual CMM determines the measurement uncertainty by a Monte Carlo method according to the GUM supplement 1 framework. This method requires the virtual-CMM software add-on that can be provided by the CMM manufacturer to the corresponding calibration lab.

To provide a software independent method, we used the second approach first introduced by Hernla [14], which has been integrated in the guideline for CMM uncertainty evaluation VDI 2617 that is released by the Association of German Engineers as supplement VDI 2617-11.

\section{Calibration of workpieces as basis for performance evaluation}

VDI 2617-11 performs a GUM-conform modeling of a CMM measurement and includes the main influence components, that are:

1. probe deviation;

2. CMM geometry deviation;

3. temperature;

4. workpiece combined with the fitting algorithms and measurement strategy (limited to Gaussian fits).

A focus of VDI 2617-11 is to offer an intensive use of GUM type B uncertainties, which avoids laborious measurements to estimate type A uncertainties.

The probe uncertainty can be easily computed either by estimating a type A uncertainty by repeated measurements of a sphere normal or deriving a type B uncertainty from the MPE of the CMM. If the MPE is given by $E=A+L / K, A / 3$ is a common approximation for the probe deviation. The CMM geometry deviation is usually always estimated as type B uncertainty from the MPE using $L / K$, where $L$ is the length of the measured feature. Estimating the geometry deviation as type A uncertainty usually leads to too small uncertainties due to unobserved influence components during the type A measurements (e.g. deflection of axes, temperature gradient, etc.).

The temperature deviation $\Delta L_{T}$ for a length measurement with given length $L$, temperature length deviations of scale $\Delta t_{W}$ and workpiece $\Delta t_{M}$, and deviations caused by the uncertainty of the coefficients of thermal expansion $\Delta L_{\alpha W}$ and $\Delta L_{\alpha M}$ with the relative uncertainties $\alpha_{W}$ and $a_{M}$ of the scale can be derived by

$$
\begin{aligned}
\Delta L_{T} & =\Delta L_{\alpha W}-\Delta L_{\alpha M}+\Delta L_{t W}-\Delta L_{t M} \\
& =L\left[\alpha_{W}\left(t_{W}-20{ }^{\circ} \mathrm{C}\right)-\alpha_{M}\left(t_{M}-20{ }^{\circ} \mathrm{C}\right)\right] .
\end{aligned}
$$

The main task to estimate the measurement uncertainty is to form a measurement equation for the CMM measurement of the feature that has to be extracted.

The features are usually the parameters $x$ (radius, inclination, etc.) of an $n$-dimensional surface that is fitted into sampled measurement points. To obtain the parameters, a homogeneous, linear equation system

$$
M x=v
$$

has to be solved, whereas $M$ is the matrix of the normal equation of the surface. $M$ can be computed by the design 


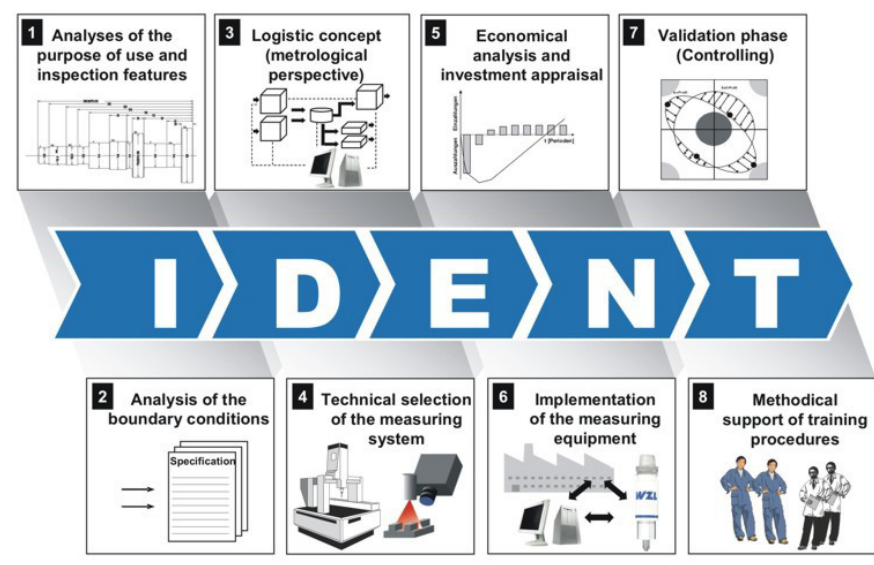

Fig. 2. IDENT method.

matrix or Jacobian $A$ for each measurement point that is

$$
M=A^{T} A \text {. }
$$

If the uncertainty of a single measurement $\sigma$-caused both by the deviation of the workpiece and the measurement system - is known, the covariance matrix of the fitting operation $S$ can be given by

$$
S=M^{-1} \cdot \sigma^{2}=Q \cdot \sigma^{2} .
$$

If $s$ is an estimate for $\sigma$, we can obtain the standard measurement uncertainty of component $i$ by the diagonal elements of matrix $Q$ with

$$
u_{i}=\sqrt{s_{i}^{2}}=\sqrt{q_{i i}} s .
$$

These equations can be formed so that the uncertainties $u_{i}$ are dependant only by $s$ and a factor $b_{i}$ that is determined by the measurement strategy, so that

$$
u_{i}=b_{i} s .
$$

For standard measurement tasks (diameter, position, inclination, etc.), a predefined set of $b_{i}$ is provided within the appendix of VDI 2611-11, while for special tasks, the measurement equation has to be formed by the user. In this work, both measurements for a standard task (radius) and a non-standard task (inner-radius distance) have been carried out (Fig. 3).

\subsection{Radius feature}

The following example shows a measurement of the broach radius feature (Fig. 3a). The radius can be computed by fitting circles into the measured points. The equation to fit a circle via linear least square regression [10] can be given by

$$
\left(x_{i}-x_{m}\right)+\left(y_{i}-y_{m}\right)+r=0 .
$$

Dividing by $r$, we have

$$
\begin{array}{r}
\left(x_{i}-x_{m}\right) / r+\left(y_{i}-y_{m}\right) / r+1=0 \\
\cos \left(\varphi_{i}\right)+\sin \left(\varphi_{i}\right)+1=0 .
\end{array}
$$

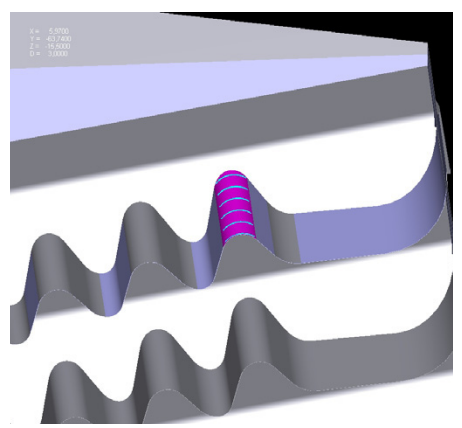

(a)

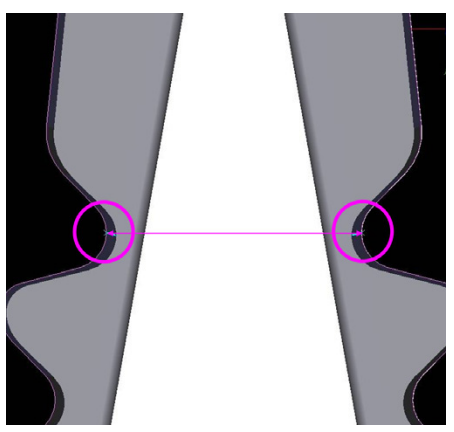

(b)

Fig. 3. Measured features of the broach: (a) inside radius, (b) distance of 2 opposite radii.

Hence, the design matrix $A$ for each point respectively angle $\varphi_{i}$ is

$$
A=\left(\begin{array}{lll}
\cos \left(\varphi_{1}\right) & \sin \left(\varphi_{1}\right) & 1 \\
\vdots & \vdots & 1 \\
\cos \left(\varphi_{n}\right) & \sin \left(\varphi_{n}\right) & 1
\end{array}\right) .
$$

If the $n$ points are taken with equal size along the entire circle, the uncertainties of radius $u_{r}$ and center of the circle $u_{m}$ are

$$
u_{m}=u_{x}=u_{y}=\sqrt{2 / 2 n} s
$$

and

$$
u_{r}=\sqrt{1 / n} s .
$$

The points that are taken from the broach do not cover the entire circle, so the uncertainty of the measured radius is larger and depends on the size of the circle sector (Fig. 5).

The measurement equation for the radius can be derived from the diameter equation that is

$$
D=D_{W}-\left(D_{T E}-D_{C}\right) \mid-\Delta_{C M M}+\Delta L_{T}
$$

with $D_{W}$ as the diameter estimation the fitting operation, $D_{T E}$ as probe error, $D_{C}$ as calibration error, $\Delta_{C M M}$ as geometry error and $\Delta L_{T}$ as the temperature error according to equation (1). Then, the radius $R$ is simply $D / 2$.

With the measurement conditions of Table 2 , the calibration has been carried out with a Zeiss UPMC 850 Carat lab CMM. Using 50 points and a covered circle sector $\varphi=100^{\circ}$ and equations (3)-(6) and (9), the corresponding factor is $b_{i}=1.11$. The uncertainty of the 
Table 2. Determined measurement conditions.

\begin{tabular}{ccc}
\hline$R=$ & 1.48 & nominal size of the radius $[\mathrm{mm}]$ \\
$\varphi=$ & 100 & covered circle sector $\left[{ }^{\circ}\right]$ \\
$A=$ & 0.8 & $A$ component of $\mathrm{MPE}=(A+L / K)[\mu \mathrm{m}]$ \\
$K=$ & 600 & $K$ component of $\mathrm{MPE}=(A+L / K)[\mu \mathrm{m}]$ \\
$U_{C}=$ & 0.2 & calibration uncertainty $[\mu \mathrm{m}]$ \\
\hline$\alpha_{M}=$ & 0 & coefficient of thermal expansion of the scale $\left[10^{-6} / \mathrm{K}\right]$ \\
$t_{M}=$ & 20.5 & average scale temperature $\left[{ }^{\circ} \mathrm{C}\right]$ \\
$\delta t_{M}=$ & 1 & maximum range of temperature deviation of the scale $[\mathrm{K}]$ \\
$\alpha_{W}=$ & 10 & coefficient of thermal expansion of the work piece $\left[10^{-6} / \mathrm{K}\right]$ \\
$t_{W}=$ & 20.5 & average work piece temperature $\left[{ }^{\circ} \mathrm{C}\right]$ \\
$\delta t_{W}=$ & 1 & maximum range of temperature deviation of the work piece $[\mathrm{K}]$ \\
\hline
\end{tabular}

Table 3. Result of the calibration of the radius feature.

\begin{tabular}{|c|c|c|c|c|c|c|}
\hline $\begin{array}{c}\text { Influance } \\
\text { quantity } \\
X_{i}\end{array}$ & $\begin{array}{c}\text { Method } \\
m_{i}\end{array}$ & $\begin{array}{l}\text { No. of points/ } \\
\text { distribution } \\
\text { type } \\
n_{i} \\
\end{array}$ & $\begin{array}{l}\text { Std. dev/error } \\
\text { range } \\
s_{i} / a_{i} \\
\end{array}$ & $\begin{array}{c}\text { Measurement } \\
\text { strategy/ } \\
\text { distribution } \\
b_{i} \\
\end{array}$ & $\begin{array}{c}\text { Sensitvity } \\
\text { coefficients } \\
c_{i} \\
\end{array}$ & $\begin{array}{l}\text { Uncertainty } \\
\quad[\mu \mathrm{m}] \\
u_{i}(y) \\
\end{array}$ \\
\hline$D_{W}$ & $\mathrm{~B}$ & 50 & 1.00 & 1.11 & 1.00 & 1.11 \\
\hline$D_{T E}$ & B & 15 & 0.50 & 0.77 & 1.00 & 0.39 \\
\hline$D_{C}$ & B & Normal & 0.20 & 0.50 & 1.00 & 0.10 \\
\hline$\Delta L_{C M M}$ & B & Normal & 0.00 & 0.50 & 1.00 & 0.00 \\
\hline$\Delta L_{\alpha M}$ & B & Rect & 0.00 & 0.58 & 0.00 & 0.00 \\
\hline$\Delta L_{\alpha W}$ & B & Rect & 2.00 & 0.58 & 0.00 & 0.00 \\
\hline$\Delta L_{t M}$ & B & Rect & 1.00 & 0.58 & 0.00 & 0.00 \\
\hline$\Delta L_{t W}$ & $\mathrm{~B}$ & Rect & 1.00 & 0.58 & 0.01 & 0.01 \\
\hline \multicolumn{7}{|c|}{$\begin{array}{l}\text { Standard uncertainty of the measurand: } u(y)=1.2 \\
\text { Coverage factor: } k=2.00 \\
\text { Extended measurement uncertainty }(P=95 \%): U=\mathbf{2 . 4}\end{array}$} \\
\hline
\end{tabular}

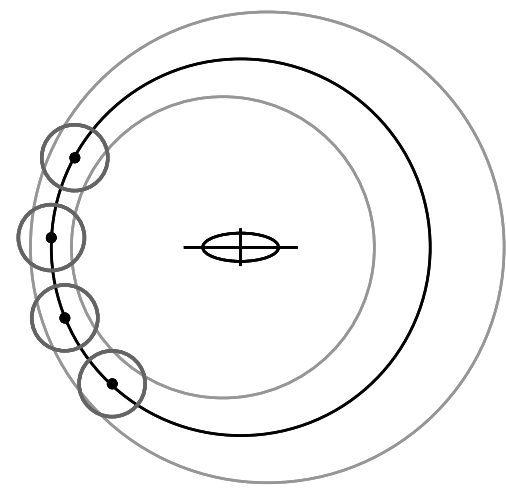

Fig. 4. Uncertainty of measured points that are not equally distributed around a circle.

calibration of the radius feature $u_{\text {rad }}$ can be computed by $u_{\text {rad }}=u_{D} / 2=2.4 \mu \mathrm{m} / 2=1.2 \mu \mathrm{m}$ according to Table 3 .

The computation of the uncertainty has been done out automatically using Matlab. Since the measurement equation (12) is taken from VDI 2617-11, the user has only to specify the measurement conditions according to Table 2.

\subsection{Inside-radius distance feature}

The second task is to perform a calibration for the insideradius distance feature (Fig. 3b). Every two of the opposing notches can modeled as two opposing sheared cylinders
(Fig. 5). The minimum distance of the two notches is

$$
\begin{gathered}
L=\mid X_{1}-\Delta X_{T 1}-\Delta R_{T 1}-W_{1}-R_{1} \\
-\left(X_{1}-\Delta X_{T 1}-\Delta R_{T 1}-W_{1}-R_{1}\right) \mid \\
-D_{C}+\Delta_{C M M}+\Delta L_{T} .
\end{gathered}
$$

$X_{1}$ denotes the center point of the fitted circle, $\Delta X_{T 1}$ the error of the center point, $R_{1}$ the radius of the fitted circle, $\Delta R_{1}$ the error of the radius and $W_{1}$ the error of the angles of the sheared cylinders. For the second cylinder, all the components $\square_{2}$ are defined respectively.

Even if this example of a non-standard measurement task is quite simple, the effort to determine and verify a correct measurement equation is significant and cannot be done by workers and engineers that are not experts in metrology and uncertainty management. Using equation (13) and again Table 2, the uncertainty of the notch distance can be computed (Tab. 4).

Hence, the standard measurement uncertainty of the feature is $u_{\text {dist }}=1.833 \mu \mathrm{m}$.

\section{Performance assessment according to ISO 14253-1 and VDA 5 using ISO 15530-3}

To estimate the uncertainty of the measurement device with unknown measurement equation, the CMM calibrated broach is used with uncertainties $u_{\text {rad }}$ and $u_{\text {dist }}$ 
Table 4. Result of the calibration of the inside-radius distance.

\begin{tabular}{|c|c|c|c|c|c|c|}
\hline $\begin{array}{l}\text { Influance } \\
\text { quantity } \\
\quad X_{i} \\
\end{array}$ & $\begin{array}{c}\text { Method } \\
m_{i}\end{array}$ & $\begin{array}{l}\text { No. of points/ } \\
\text { distribution } \\
\text { type } \\
n_{i} \\
\end{array}$ & $\begin{array}{l}\text { Std. dev/error } \\
\text { range } \\
s_{i} / a_{i} \\
\end{array}$ & $\begin{array}{l}\text { Measurement } \\
\text { strategy/ } \\
\text { distribution } \\
b_{i} \\
\end{array}$ & $\begin{array}{c}\text { Sensitvity } \\
\text { coefficients } \\
c_{i} \\
\end{array}$ & $\begin{array}{l}\text { Uncertainty } \\
{[\mu \mathrm{m}]} \\
u_{i}(y)\end{array}$ \\
\hline$X_{1}$ & $\mathrm{~B}$ & 50 & 1.00 & 0.93 & 1.00 & 0.93 \\
\hline$R_{1}$ & B & 50 & 1.00 & 0.82 & 1.00 & 0.82 \\
\hline$W_{1}$ & B & 50 & 1.00 & 0.11 & 1.00 & 0.11 \\
\hline$X_{2}$ & B & 50 & 1.00 & 0.93 & 1.00 & 0.93 \\
\hline$R_{2}$ & B & 50 & 1.00 & 0.82 & 1.00 & 0.82 \\
\hline$W_{2}$ & B & 50 & 1.00 & 0.11 & 1.00 & 0.11 \\
\hline$\Delta R_{T 1}$ & B & 15 & 0.50 & 0.39 & 0.50 & 0.10 \\
\hline$\Delta R_{T 2}$ & B & 15 & 0.50 & 0.39 & 0.50 & 0.10 \\
\hline$\Delta X_{T 1}$ & B & 15 & 0.50 & 0.66 & 1.00 & 0.33 \\
\hline$\Delta X_{T 2}$ & B & 15 & 0.50 & 0.66 & 1.00 & 0.33 \\
\hline$D_{C}$ & B & Normal & 0.20 & 0.50 & 1.00 & 0.10 \\
\hline$\Delta L_{C M M}$ & B & Normal & 0.02 & 0.50 & 1.00 & 0.01 \\
\hline$\Delta L_{\alpha_{M}}$ & B & Rect & 0.00 & 0.58 & 0.01 & 0.00 \\
\hline$\Delta L_{\alpha_{W}}$ & B & Rect & 2.00 & 0.58 & 0.01 & 0.01 \\
\hline$\Delta L_{t M}$ & B & Rect & 1.00 & 0.58 & 0.00 & 0.00 \\
\hline$\Delta L_{t W}$ & $\mathrm{~B}$ & Rect & 1.00 & 0.58 & 0.16 & 0.09 \\
\hline \multicolumn{7}{|c|}{$\begin{array}{l}\text { Standard uncertainty of the measurand: } u(y)=1.83 \\
\text { Coverage factor: } k=2.00 \\
\text { Extended measurement uncertainty }(P=95 \%): U=\mathbf{3 . 6 6}\end{array}$} \\
\hline
\end{tabular}

and the calibration results $x_{c a l}$ that are computed according to the method of Chapter 3.

According to ISO 15530-3, the two features of the calibrated broach have to be measured at least $n=20$ times by different operators, at different times, and at different temperature conditions. From these measurements, a standard uncertainty $u_{p}$ for the repeated measurements $y_{i}$ of the measurement process is computed, which is simply the standard deviation using $\bar{y}=\frac{1}{n} \sum_{i=1}^{n} y_{i}$ and

$$
u_{p}=\sqrt{\frac{1}{1-n} \sum_{i=1}^{n}\left(y_{i}-\bar{y}\right)^{2}} .
$$

In ISO 15530-3, the uncertainty for the temperature deviation of an object with temperature $t$, uncertainty of the thermal expansion $u_{\alpha}$ and the object length $L$ is proposed by

$$
u_{w}=\left(t-20{ }^{\circ} \mathrm{C}\right) u_{\alpha} L .
$$

A more precise method that is used in this work also comprises the thermal uncertainties of the scale using equation (1):

$$
\begin{aligned}
u_{\Delta L_{T}}= & L\left(\left(\alpha_{w} u_{t W}\right)^{2}+\left(\alpha_{M} u_{t M}\right)^{2}\right. \\
& \left.+\left[\left(T-20^{\circ} \mathrm{C}\right) u_{\alpha M}\right]^{2}+\left[\left(T-20^{\circ} \mathrm{C}\right) u_{\alpha W}\right]^{2}\right)^{\frac{1}{2}}
\end{aligned}
$$

Using the calibration results $x_{c a l}$, the bias of the repeated measurement is

$$
b=\bar{y}-x_{c a l} .
$$

With the uncertainty of the calibration $u_{c a l}=u_{\text {rad }}$ or $u_{c a l}=u_{d i s t}$, and a coverage factor $k$, the combined

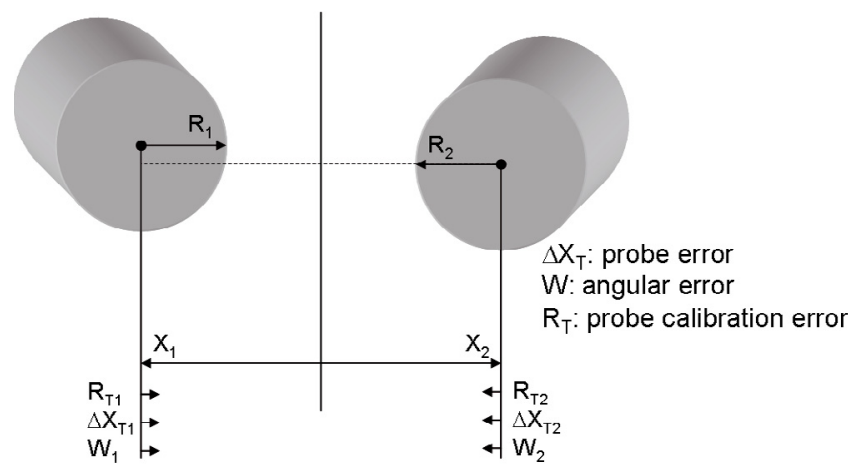

Fig. 5. Derivation of the measurement equation for the radiusdistance feature.

extended measurement uncertainty $U$ is

$$
U=k \sqrt{u_{\Delta L_{T}}^{2}+u_{c a l}^{2}+u_{p}^{2}}+|b| .
$$

To verify the conformance of a broach according to ISO14253-1, it has to be checked, whether the measured sizes lie within the remaining tolerance that is the basic tolerance reduced by the extended uncertainty $U$ at both tolerance limits (Fig. 6).

This method does not determine upper boundaries for the uncertainty of the measurement system, which can yield to high $\alpha$ - and $\beta$-errors for the conformance decision. More effective is to set upper limits to the measurement uncertainty. A common upper limit is defined by the automotive guideline VDA 5 by

$$
\frac{U}{T} \leqslant 0.1 \ldots 0.2
$$




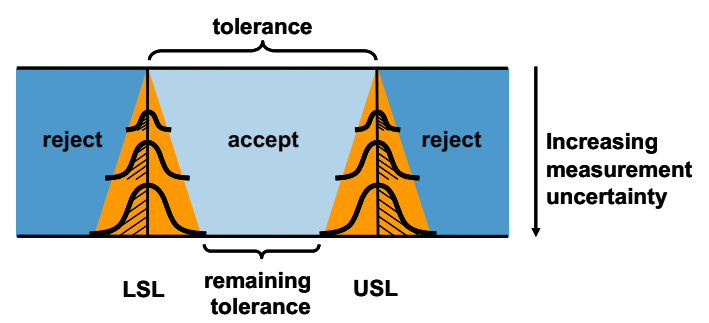

Fig. 6. Conformance according to ISO 14253-1.

so that the extended uncertainty $U$ is a factor 10 to 20 lower than the specified tolerance.

\section{Conclusions}

Conformance assessment of measurement processes is increasingly required and implemented in the industrial use of metrology. One particular challenge in this respect is the computation of measurement uncertainties for complex measuring processes. VDI 2617-11 in combination with ISO 15530-3 and ISO 14253-1 provides a software independent and consistent scientific framework for estimating measurement uncertainties for measurement devices with unknown measurement behavior with the need of additional calibration measurements of a master workpiece. For standard measurement task, this process can be fully automated using predefined measurement equations of the VDI 2617-11 appendix.

On the other hand, modeling non-standard measurement tasks according to VDI 2617-11 calls for a high level of expert knowledge. Even then, there remains a risk of neglecting uncertainty influences and hence producing an incorrect estimate of measurement uncertainty. The effort needed for mathematical formulation of the error model and derivation of the more complex equations is often unmanageable.

Acknowledgements. We would like to thank Susanne Nisch for performing the calibration and their continuous support with all aspects of coordinate metrology. Parts of this work were funded by the German Academic Exchange Service (DAAD). We would also like to thank the German Research Foundation DFG for the support and the funding of the depicted research within the Cluster of Excellence "Integrative Production Technology for High-Wage Countries".

\section{References}

1. ISO 9000, Quality management systems - Fundamentals and vocabulary (2005)

2. ISO 9001, Quality management systems - Requirements (2000)

3. ISO 10012, Measurement management systems Requirements for measurement processes and measuring equipment (2003)

4. ISO/TS 16949, Quality management systems - Particular requirements for the application of ISO 9001:2008 for automotive production and relevant service part organizations (2009)

5. AIAG, Measurement System Analysis (MSA), 3rd edn. (2002)

6. Verband der Automobilindustrie, VDA 5 - Qualitätsmanagement in der Automobilindustrie, Prüfprozesseignung, 1st edn. (2003)

7. ISO 13005, Guide to the Expression of Uncertainty in Measurement (1999)

8. DIN EN ISO 14253-1, Geometrical product specifications (GPS) - Inspection by measurement of workpieces and measuring equipment - Part 1: Decision rules for proving conformance or non-conformance with specifications (1999)

9. R. Schmitt, P. Fritz, et al., Estimation of Complex Measurements Systems by Statistical Simulation (in German), VDI Tagung Messunsicherheit praxisgerecht bestimmen (2008)

10. R. Schmitt, P. Fritz, et al., Evaluating the Uncertainty of Complex Measurement Systems, 14e Congress de Metrologie (2009)

11. ISO 15530-3, Geometrical Product Specifications (GPS) - Coordinate measuring machines (CMM): Technique for determining the uncertainty of measurement - Part 3: Use of calibrated workpieces or standards (2004)

12. S. Driessen, B. Engelmann, B. Dietrich, Mit Planung ans Ziel - Das Strategietool IDENT für einen optimierten Einsatz von Fertigungsmesstechnik unter Qualitäts- und Kostenkriterien, Qualität und Zuverlässigkeit - Sonderheft Festschrift zur Emeritierung von Prof. Pfeifer (2004), pp. $19-20$

13. F. Waeldele, H. Schwenke, Automated Calculation of Measurement Uncertainties on CMMs - Towards industrial application, Technisches Messen 69, 550-557 (2002)

14. M. Hernla, Task Specific Uncertainty in Coordinate Measurement, Technisches Messen 64, 286-293 (1997)

15. A. B. Forbes, Nonlinear Least Squares and Bayesian Inference, Adv. Math. Comput. Tools Metrol. Testing 8, 104-112 (2009) 